\section{P653 MODELING THE IMPACT OF PARTIALLY EFFICACIOUS GONORRHEA VACCINES}

${ }^{1}$ Shaheen Seedat*, ${ }^{2}$ Laith Abu-Raddad. 'Weill Cornell Medicine-Qatar, Infectious Disease Epidemiology Group, Doha, Qatar; ${ }^{2}$ Weill Cornell Medicine-Qatar, Doha, Qatar

10.1136/sextrans-2019-sti.721

Background Gonorrhea is one of the most common sexually transmitted infections. Current control measures are inadequate and are threatened by the emergence of gonococcal antimicrobial resistance. This emerging challenge calls for a vaccine which will reduce the circulation and transmission of this infection, and thus emergence of drug-resistant strains in the population.

Methods A novel deterministic compartmental mathematical model of the heterosexual transmission of Neisseria gonorrheae was constructed to assess the impact of a pre-exposure (prophylactic) vaccine.

Results Catch-up vaccination with a prophylactic vaccine introduced in 2020, with vaccine efficacy in reducing susceptibility of $50 \%$ and vaccine coverage of $80 \%$ at 2030 , reduced gonorrhea prevalence by $29 \%$ by $2030,34 \%$ by 2040 , and $37 \%$ by 2050 . The number of vaccinations needed to avert one infection was 31 in 2030, 24 in 2040, and 13 in 2050. Through age group prioritization, the number of vaccinations needed to avert one infection (in 2030) ranged from 24 for the 15-19 years age group, to 50 for the 45-49 age group. Through risk group prioritization (also in 2030), prioritizing the highest sexual risk group (such as female sex workers) was most effective with only 1 vaccination needed per infection averted. Meanwhile, for the lowest sexual risk group (general population), 110 vaccinations were needed per infection averted.

Conclusion Even a partially efficacious gonorrhea vaccine can considerably reduce the prevalence of infection. Vaccine effectiveness is optimized by targeting high sexual risk groups and young individuals.

Disclosure No significant relationships.

\section{P654 CLONAL SPREAD OF AZITHROMYCIN RESISTANT NEISSERIA GONORRHOEAE IN CANADA, 2014-2017}

${ }^{1}$ Irene Martin*, ${ }^{1}$ Pam Sawatzky, ${ }^{2}$ Brigitte Lefebvre, ${ }^{3}$ Vanessa Allen, ${ }^{4}$ Prenilla Naidu, ${ }^{5}$ Linda Hoang, ${ }^{6}$ Jessica Minion, ${ }^{7}$ Daivd Haldane, ${ }^{8}$ Mike Mulvey. ${ }^{1}$ Public Health Agency of Canada, National Microbiology Laboratory, Winnipeg, Canada; ${ }^{2}$ Laboratoire Santé Publique du Québec, Montréal, Canada; ${ }^{3}$ Public Health Ontario, Toronto, Canada; ${ }^{4}$ Provincial Laboratory for Public Health, Edmonton, Canada; ${ }^{5}$ British Columbia Centres for Disease Control, Vancouver, Canada; ${ }^{6}$ Saskatchewan Health Authority, Regina Qu'appelle Health Region, Regina, Canada; ${ }^{7}$ Provincial Public Health Laboratory Network of Nova Scotia, Halifax, Canada; ${ }^{8}$ Public Health Agency of Canada, Winnipeg, Canada

10.1136/sextrans-2019-sti.722

Background Neisseria gonorrhoeae have acquired resistance to many antimicrobials including third generation cephalosporins and azithromycin, the current co-therapy recommended by the Canadian STI guidelines for gonorrhea treatment. Resistance to azithromycin and molecular sequence types were determined for N. gonorrhoeae circulating in Canada.

Methods From 2014-2017, N. gonorrhoeae isolates and data collected by Canadian provincial public health laboratories was submitted to the National Microbiology Laboratory $(\mathrm{N}=12,776)$ for $N$. gonorrhoeae multi-antigen sequence typing (NG-MAST) and azithromycin MIC determination (resistance
MIC $\geq 2.0 \mathrm{mg} / \mathrm{L}$ ) by agar dilution, as described by the Clinical Laboratory Standards Institute.

Results Azithromycin resistance was identified in 3.3\% (127/ 3,809), 4.7\% (198/4,190), 7.2\% (326/4,538) and 11.6\% (616/ $5,290)$ of $N$. gonorrhoeae in 2014, 2015, 2016 and 2017, respectively, a significant increase between 2014-2017 $(\mathrm{p}<0.001)$. MICs ranged from $2-\geq 256 \mathrm{mg} / \mathrm{L}$. The most common sequence types identified in the azithromycin resistant isolates include: 2014; ST10451 $(\mathrm{n}=40)$, ST10567 $(\mathrm{n}=38)$ and ST11765 $(\mathrm{n}=10) ; 2015 ;$ ST12302 $(\mathrm{n}=110)$, ST10451 $(n=34)$ and ST9047 $(n=23) ; 2016:$ ST12302 $(n=240)$, ST15750 $(n=27)$ and ST10451 $(n=10) ; 2017 ;$ ST12302 $(\mathrm{n}=375)$, ST14698 $(\mathrm{n}=119)$ and ST15750 $(\mathrm{n}=17)$. ST12302 was newly recognized in 2015 and identified in only two provinces, Quebec and Ontario, but spread to Alberta $(n=10)$ and British Columbia $(n=16)$ in 2017. ST12302 is associated with low-level azithromycin resistance $(\mathrm{MIC}=2 \mathrm{mg} / \mathrm{L})$. ST10451 emerged in 2014 in Quebec, Ontario and Alberta and was also identified in 2015-2017. ST10451 is related to ST1407 (differing by $1 \mathrm{bp}$ ) which is an internationally-recognized epidemic strain, harboring cephalosporin resistance.

Conclusion Azithromycin resistance in N. gonorrhoeae is established and spreading in Canada, increasing significantly between 2014 and 2017. This exceeds the 5\% level at which the WHO states an antimicrobial should be reviewed as an appropriate treatment. Continued surveillance of antimicrobial susceptibilities and sequence types of $N$. gonorrhoeae is necessary to identify clusters, inform treatment guidelines and mitigate the impact of resistant gonorrhea.

Disclosure No significant relationships.

\section{P655 MOLECULAR EPIDEMIOLOGY ASSOCIATED WITH RESISTANCE IN NEISSERIA GONORRHOEAE ISOLATES FROM SOUTH BRAZIL DURING 2008-2016}

${ }^{1}$ Lisléia Golfetto, ${ }^{1}$ Marcos Schörner, ${ }^{2}$ Thaís Santos, 'Jéssica Martins, ${ }^{1}$ Hanalydia Machado, ${ }^{1}$ Felipe De Rocco, ${ }^{1}$ Mara Scheffer, ${ }^{2}$ Emanuelle Da Silva, ${ }^{2}$ Nina Tobouti, ${ }^{2}$ Cássia Zoccoli, ${ }^{1}$ Maria Bazzo*. ${ }^{1}$ Federal University of Santa Catarina, Florianópolis, Brazil; ${ }^{2}$ Santa Luzia Medical Laboratory, Florianópolis, Brazil

10.1136/sextrans-2019-sti.723

Background Neisseria gonorrhoeae (NG) has an extraordinary ability to develop resistance to all antimicrobials used for its treatment. This study analysed molecular determinants of antimicrobial resistance and NG-MAST of $153 \mathrm{NG}$ isolates collected at Florianopolis metropolitan area during 2008-2016. Methods Minimal Inhibitory Concentration (MIC) was determined by agar dilution and the molecular epidemiology was evaluated by NG-MAST.

Results Resistance was observed to penicillin (PEN) (26.1\%), tetracycline (TET) (41.2\%), ciprofloxacin (CIP) (52.3\%) and azithromycin (AZT) (5.2\%). All isolates were susceptible to cefixime (CFX) and ceftriaxone (CRO). However, $8.5 \%$ of isolates had $\mathrm{MIC}=0.125 \mathrm{mg} / \mathrm{L}$ for $\mathrm{CFX}$, one $\log$ below the resistance cut-off point (EUCAST). $\beta$-lactamase production was detected in $12.4 \%$ of isolates and one of them carried the bla $a_{\text {TEM-135 }}$ allele. The American or Dutch tet $\mathrm{M}$ gene were carried by $5.2 \%$ of the isolates. Mutations in the QRDR were observed in $87.5 \%$ of isolates resistant to CIP. NG-MAST showed 64 different sequence types (STs), including 19 novel STs. ST225, ST2992, ST1582, ST338, ST1407, ST2202 and ST6827 were most prevalent. G225 genogroup was associated with resistance to CIP $(p<0.001)$, PEN $(p=0.016)$ and TET 